\title{
Association of COVID-19 Infection and Purpura Fulminans
}

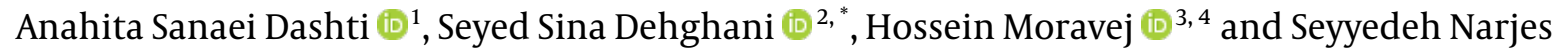 \\ Abootalebi $^{5}$ \\ ${ }^{1}$ Professor Alborzi Clinical Microbiology Research Center, Shiraz, Iran \\ ${ }^{2}$ School of Medicine, Shiraz University of Medical Sciences, Shiraz, Iran \\ ${ }^{3}$ Department of Pediatric Endocrinology, School of Medicine, Shiraz University of Medical Sciences, Shiraz, Iran \\ ${ }^{4}$ Neonatal Research Center, Shiraz University of Medical Sciences, Shiraz, Iran \\ ${ }^{5}$ Department of Pediatrics, School of Medicine, Shiraz University of Medical Sciences, Shiraz, Iran \\ "Corresponding author: School of Medicine, Shiraz University of Medical Sciences, Shiraz, Iran. Email: dehghani.sina77@gmail.com
}

Received 2020 September 29; Revised 2020 November 25; Accepted 2020 December 01.

\begin{abstract}
Coronavirus disease 2019 (COVID-19) was officially declared as a pandemic on March 11, 2020. Although most patients with COVID-19 manifest fever and respiratory tract symptoms, extrapulmonary and atypical presentations, such as gastrointestinal, neurologic, and cardiovascular involvement as well as thromboembolic events have been reported amongst COVID-19 patients. Herein, we aim to describe a COVID-19 patient who progressed to purpura fulminans.
\end{abstract}

Keywords: Children, COVID-19, Purpura Fulminans

\section{Introduction}

Severe acute respiratory syndrome coronavirus 2 (SARS-COV-2) is a novel virus that has affected millions of individuals across the world. Coronavirus disease 2019 (COVID-19) was officially declared as a pandemic on March 11, 2020 (1-3). This disease most frequently is manifested as viral pneumonia characterized by symptoms such as fever, dyspnea, and cough. However, extrapulmonary and atypical presentations, such as gastrointestinal, neurologic, and cardiovascular involvement, as well as thromboembolic events, have been reported amongst COVID-19 patients (4-7). Herein, we present a patient referred to Namazi Hospital, Shiraz, Iran, with an impression of gastroenteritis whose COVID-19 PCR turned out to be positive and progressed to purpura fulminans (PF).

\section{Case Presentation}

A3-year-old, full-term, and fully immunized female was referred to Namazi Hospital with a history of fever, diarrhea, nausea, and vomiting since five days prior to admission. The patient was very ill. However, her mother reported no symptoms regarding respiratory infections, and she had no signs of respiratory tract involvement. The patient had no definite COVID-19 exposure history.

On physical examination, her pulses were not detected. Thus, her blood pressure could not be measured. The patient's respiratory rate was 38 per minute, and the temperature was $38.2^{\circ} \mathrm{C}$. Her arterial oxygen saturation was 74\% in room air. The patient was lethargic and had a decreased level of consciousness. The patient's height and weight were $90 \mathrm{~cm}$ and $8 \mathrm{~kg}$, respectively. Some ecchymotic lesions were all over the body, especially in sites where blood was drawn. Both hands and feet were cold, pulseless, mottled, and covered by ecchymosis, particularly in more distal parts (Figure 1). Her fingers were cyanotic, and capillary refill time was delayed for more than 3 seconds. No other abnormal signs were detected in physical examination. Table 1 showed the laboratory data of the patient.

Due to the COVID-19 outbreak, the PCR of COVID-19 was done on samples of the pharynx and nasopharynx of the patient, which turned positive. Spiral HRCT of lungs showed patchy ground-glass and patchy consolidations in the upper and lower lobes of both lungs, which was in favor of COVID-19 infection (Figure 2). The patient had undergone echocardiography, which revealed a mildly dilated 


\begin{tabular}{|c|c|c|c|c|}
\hline Lab Data & On Admission & 1 Day After Admission & 2 Days After Admission & 1 Week After Admission \\
\hline $\mathrm{WBC}, / \mathbf{m m}^{3}$ & 1900 & 1900 & 1300 & 1900 \\
\hline $\mathrm{Hb}, \mathbf{g m} / \mathbf{d L}$ & 9.4 & 7.1 & 6.6 & 4.8 \\
\hline MCV, fL & 72.7 & 71.2 & 71.4 & 74.9 \\
\hline Platelet, $/ \mathrm{mm}^{3}$ & 37,000 & 38,000 & 89,000 & 216,000 \\
\hline PT, sec & 21.9 & 26.4 & 22.2 & 20.4 \\
\hline INR, index & 1.62 & 1.96 & 1.64 & 1.51 \\
\hline PTT, sec & 28.4 & 120 & 41.4 & 39 \\
\hline Blood sugar, mg/dL & 30 & & & \\
\hline B.U.N, mg/dL & 15 & & & 25 \\
\hline $\mathrm{Cr}, \mathrm{mg} / \mathrm{dL}$ & 0.7 & & & 0.3 \\
\hline $\mathrm{Na}, \mathbf{m E q} / \mathbf{L}$ & 151 & 154 & 165 & 158 \\
\hline $\mathbf{K}, \mathbf{m E q} / \mathbf{L}$ & 3.2 & 2.4 & 3.5 & 5.5 \\
\hline $\mathrm{Ca}, \mathrm{mg} / \mathrm{dL}$ & 7.6 & 7.7 & & 7.8 \\
\hline$P, \mathbf{m g} / \mathrm{dL}$ & & & & 2.1 \\
\hline Mg, mg/dL & 2.3 & & & 1.4 \\
\hline Uric acid, mg/dL & & & 3.1 & \\
\hline AST, IU/L & & & 26 & 75 \\
\hline ALT, IU/L & & & 39 & 68 \\
\hline ALK.P, IU/L & & & 235 & 191 \\
\hline Total bilirubin, mg/dL & & & 0.6 & 0.6 \\
\hline Direct bilirubin, mg/dL & & & 0.3 & 0.3 \\
\hline Total protein, g/dL & & & 4.2 & 4.7 \\
\hline Albumin, g/dL & & & 2.4 & 2.9 \\
\hline LDH, IU/L & & & 812 & 1810 \\
\hline ESR, $\mathbf{m m} / \mathbf{h}$ & & 13 & 50 & \\
\hline CPK, IU/L & & & & 163 \\
\hline CRP, mg/L & & $>150$ & $>150$ & 136 \\
\hline Ferritin, ng/mL & & & 510.7 & \\
\hline Procalcitonin, ng/mL & & & & 2.22 \\
\hline Urine protein & Negative & & $2+$ & Negative \\
\hline Urine $\mathrm{Hb}$ & $1+$ & & $1+$ & Negative \\
\hline Random urine $\mathrm{Cr}$, mg & & & 42 & \\
\hline Urine random $\mathrm{Na}, \mathrm{mEq} / \mathrm{L}$ & & & 13.6 & \\
\hline Blood culture & & & & No growth \\
\hline Urine culture & & & & No growth \\
\hline
\end{tabular}

${ }^{a}$ Of note is the significantly prolonged PT, INR, and PTT. 

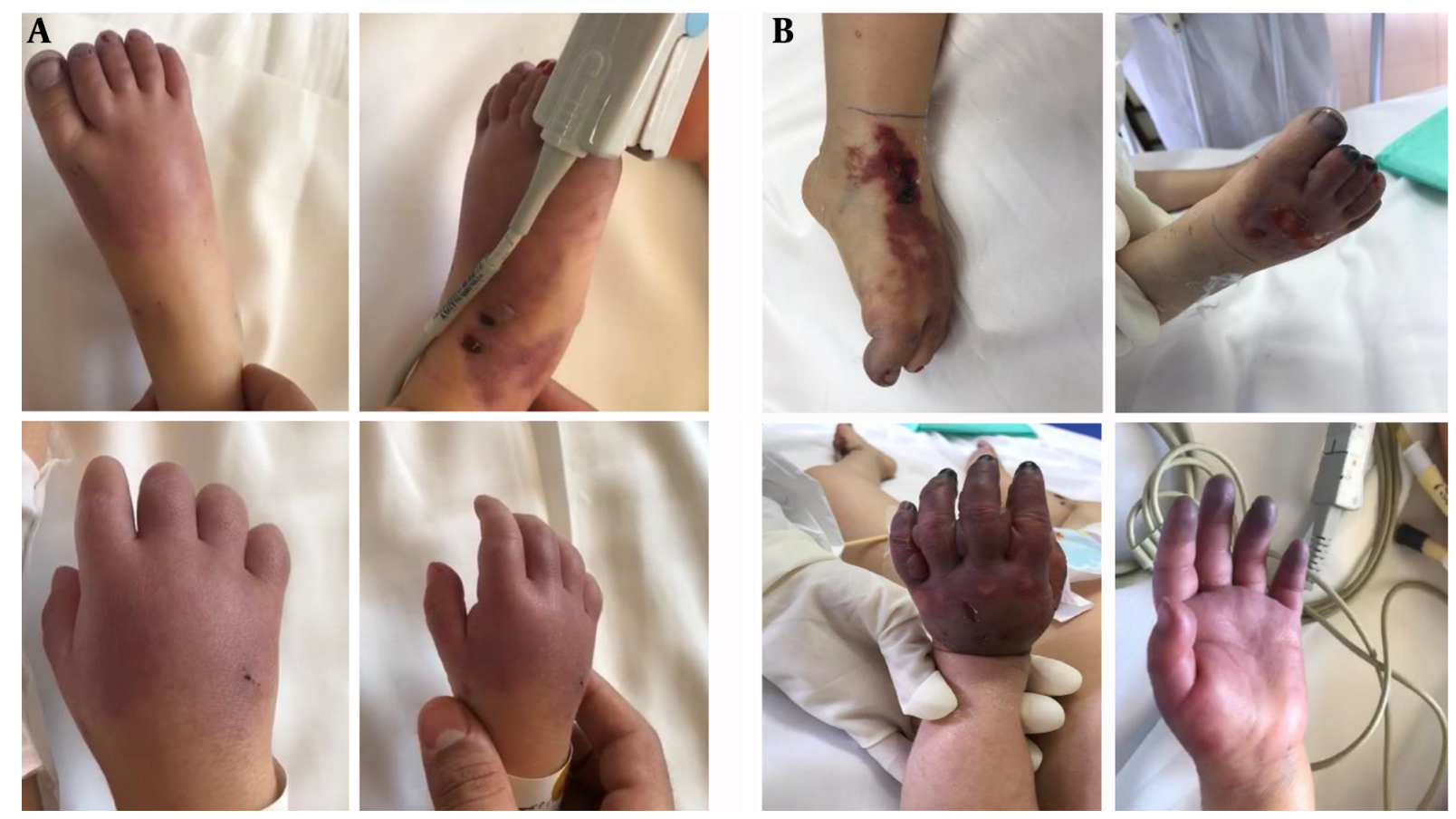

Figure 1. Presentation of PF of the patient: A, On admission; B, during her course of hospitalization

left ventricle and good biventricular function. On the second day after admission, a peripheral blood smear was taken, which showed leukopenia (lymphocyte dominant), hypochromic RBCs, moderate Burr cell count, a few acanthocytes, and severe thrombocytopenia as well as a few large platelets. Based on the pediatric hematologist and pediatric ICU specialists' recommendations, vasopressors and management of PF were started promptly. The patient was transferred to the pediatric ICU after a few hours.

Unfortunately, despite all efforts, due to the severity of the disease, the patient passed away a few days later.

\section{Discussion}

$\mathrm{PF}$ is a rare, life-threatening disorder of acute onset skin lesions due to disseminated intravascular coagulopathy (DIC) and small vessel thrombosis of the dermis, which is characterized by hemorrhagic infarction of skin (8). DIC triggers systemic activation of blood coagulation resulting in excessive blood clotting, which leads to endothelial injury. As the condition progresses, clotting factors and platelets are consumed in widespread intravascular coagulation, which can result in severe bleeding (9). Although bacteria such as Neisseria meningitidis and Streptococcus pneumoniae are the most common microorganisms that cause PF, viruses can rarely cause it (10). Shah et al. (11) explained a rare association of purpura fulminans with West Nile Virus infection. Thomson et al. (12) reported varicella zoster infection in a patient that progressed to PF. Antibodies against protein $S$ occur, and protein $S$ deficiency induced by varicella-zoster infection can be the etiology of PF in this patient $(12,13)$. According to research that assessed maternal deaths due to infectious cause, there was a significant association between the influenza $A(\mathrm{H} 1 \mathrm{~N} 1)$ virus and purpura fulminans (14). Clinical patterns of COVID-19 patients with DIC are varied, from petechial to acro-ischemia presentations. Hypercoagulable states in DIC are associated with these cutaneous manifestations. Purpura fulminans is a combination of hemorrhagic and thrombotic presentations, which can occur in severe COVID-19 patients (15, 16).

The patient was visited on September 19, 2020. To the best of our knowledge, up until now, no published COVID19 case with PF is presented in the literature, and this appears to be the first reported case of COVID-19 and PF. 

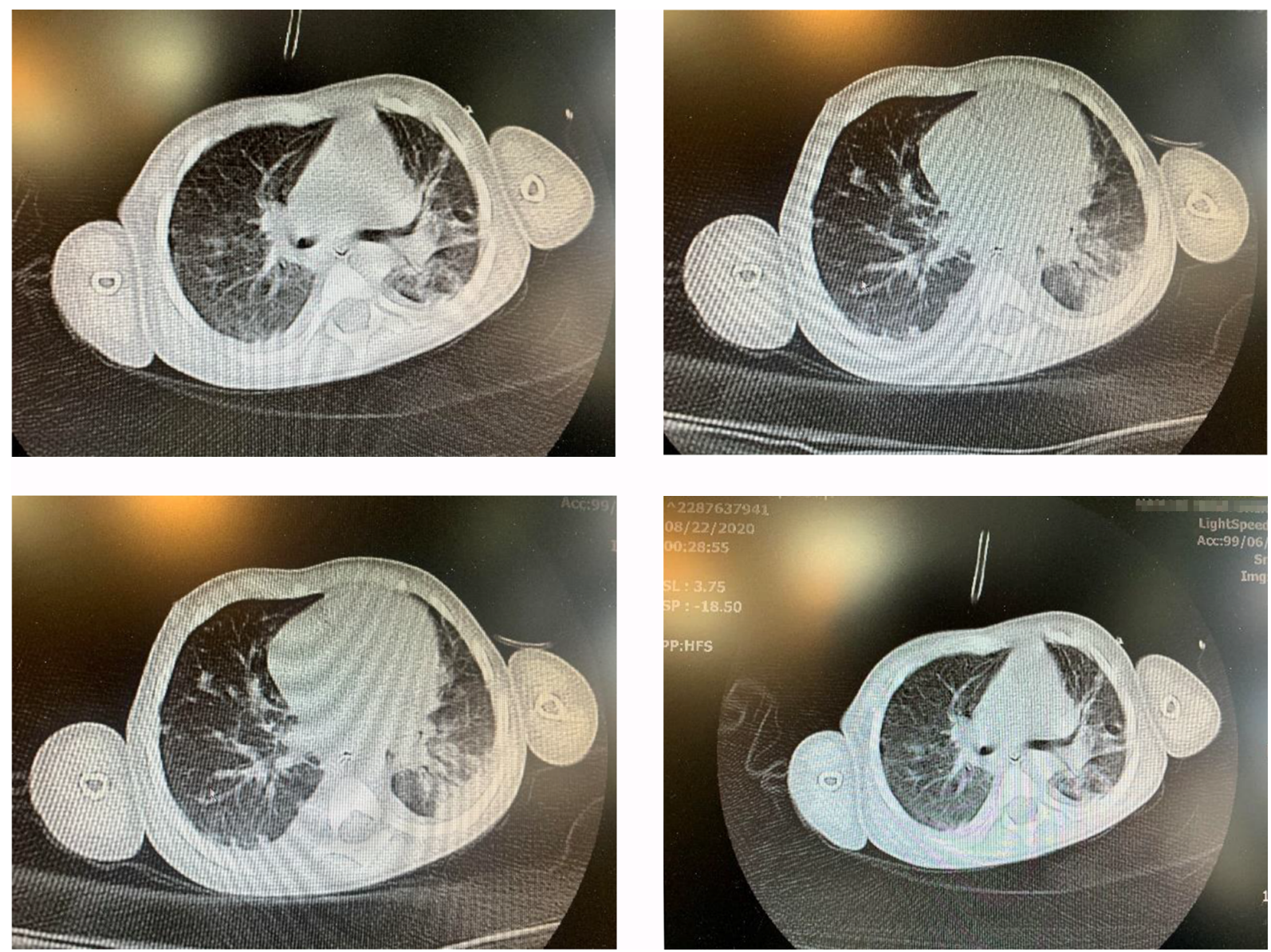

Figure 2. Chest computer tomography revealing patchy ground-glass and patchy consolidation involvements

\section{Footnotes}

Authors' Contribution: Study concept and design: Anahita Sanaei Dashti. Acquisition of data and drafting of the manuscript: Seyed Sina Dehghani. Clinician responsible for patient's treatment: Hossein Moravej and Seyyedeh Narjes Abootalebi

Conflict of Interests: The authors have no conflict of interest.

Funding/Support: No funding has been received.

Informed Consent: Informed consent has been taken from the patient's parent.

\section{References}

1. Tape C, Byrd KM, Aung S, Lonks JR, Flanigan TP, Rybak NR. COVID-19 in a Patient Presenting with Syncope and a Normal Chest X-ray. $R I$ Med J (2013). 2020;103(3):50-1. [PubMed: 32226962]. [PubMed Central: PMC7385703].
2. Bose S, Adapa S, Konala VM, Gopalreddy H, Sohail S, Naramala S, et al. Atypical Presentation of Novel Coronavirus Disease 2019 in a Peritoneal Dialysis Patient. J Investig Med High Impact Case Rep. 2020;8:2324709620931240. doi: 10.1177/2324709620931238. [PubMed: 32525402]. [PubMed Central: PMC7290252].

3. Rismanbaf A. Potential Treatments for COVID-19; a Narrative Literature Review. Arch Acad Emerg Med. 2020;8(1). e29. [PubMed: 32232214]. [PubMed Central: PMC7085862].

4. Rajewska A, Mikolajek-Bedner W, Lebdowicz-Knul J, Sokolowska M, Kwiatkowski S, Torbe A. COVID-19 and pregnancy - where are we now? A review. J Perinat Med. 2020;48(5):428-34. doi: 10.1515/jpm-2020-0132. [PubMed: 32452195].

5. Cheung KS, Hung IFN, Chan PPY, Lung KC, Tso E, Liu R, et al Gastrointestinal Manifestations of SARS-CoV-2 Infection and Virus Load in Fecal Samples From a Hong Kong Cohort: Systematic Review and Meta-analysis. Gastroenterology. 2020;159(1):81-95. doi: 10.1053/j.gastro.2020.03.065. [PubMed: 32251668]. [PubMed Central: PMC7194936].

6. Eshak N, Abdelnabi M, Ball S, Elgwairi E, Creed K, Test V, et al Dysautonomia: An Overlooked Neurological Manifestation in a Critically ill COVID-19 Patient. Am J Med Sci. 2020;360(4):427-9. doi 
10.1016/j.amjms.2020.07.022. [PubMed: 32739039]. [PubMed Central: PMC7366085].

7. Su YB, Kuo MJ, Lin TY, Chien CS, Yang YP, Chou SJ, et al. Cardiovascular manifestation and treatment in COVID-19. J Chin Med Assoc. 2020;83(8):704-9. doi: 10.1097/JCMA.0000000000000352. [PubMed: 32433342]. [PubMed Central: PMC7493780].

8. Aroor S, Varma C, Mundkur SC. Purpura fulminans in a child: a case report. J Clin Diagn Res. 2012;6(10):1812-3. doi: 10.7860/JCDR/2012/4339.2618. [PubMed: 23373061]. [PubMed Central: PMC3552237].

9. Piparsania S, Rajput N, Singh K, Zade P, Joshi M. Purpura Fulminans in Infantile Streptococcal Septicemia. Surg Sci. 2011;2(10):496-8. doi: 10.4236/ss.2011.210109.

10. Contou D, Sonneville R, Canoui-Poitrine F, Colin G, Coudroy R, Pene $F$, et al. Clinical spectrum and short-term outcome of adult patients with purpura fulminans: a French multicenter retrospective cohort study. Intensive Care Med. 2018;44(9):1502-11. doi: 10.1007/s00134-0185341-3. [PubMed: 30128591].

11. Shah S, Fite LP, Lane N, Parekh P. Purpura fulminans associated with acute West Nile virus encephalitis. J Clin Virol. 2016;75:1-4. doi: 10.1016/j.jcv.2015.11.034. [PubMed: 26686320].

12. Thomson JJ, Retter A, Hunt BJ. Novel management of post varicella purpura fulminans owing to severe acquired protein $S$ deficiency. Blood Coagul Fibrinolysis. 2010;21(6):598-600. doi: 10.1097/MBC.0b013e32833c2b52. [PubMed: 20625278].

13. Josephson C, Nuss R, Jacobson L, Hacker MR, Murphy J, Weinberg A, et al. The varicella-autoantibody syndrome. Pediatr Res. 2001;50(3):34552. doi: 10.1203/00006450-200109000-00009. [PubMed: 11518821].

14. Rigouzzo A, Tessier V, Zieleskiewicz L. [Maternal deaths due to infectious cause, results from the French confidential enquiry into maternal deaths, 2010-2012]. Gynecol Obstet Fertil Senol. 2017;45(12S):S48-53. French. doi: 10.1016/j.gofs.2017.10.016. [PubMed: 29108905].

15. Almutairi N, Schwartz RA. COVID-19 with dermatologic manifestations and implications: An unfolding conundrum. Dermatol Ther. 2020;33(5). e13544. doi: 10.1111/dth.13544. [PubMed: 32385869]. [PubMed Central: PMC7261983].

16. Thornsberry LA, LoSicco KI, English J3. The skin and hypercoagulable states. J Am Acad Dermatol. 2013;69(3):450-62. doi: 10.1016/j.jaad.2013.01.043. [PubMed: 23582572]. 